\title{
Transcranial photobiomodulation enhances visual working memory capacity in humans
}

${ }^{a}$ State Key Laboratory of Cognitive Neuroscience and Learning \&IDG/McGovern Institute for Brain Research, Beijing Normal University, Beijing, China

${ }^{\mathrm{b}}$ Center for Cognition and Neuroergonomics, State Key Laboratory of Cognitive Neuroscience and Learning, Beijing Normal University at Zhuhai, China

${ }^{\mathrm{c}}$ School of Systems Science, Beijing Normal University, Beijing, China

${ }^{\mathrm{d}}$ Department of Bioengineering, the University of Texas at Arlington, Arlington, Texas, USA

${ }^{\mathrm{e}}$ Centre for Human Brain Health, School of Psychology, University of Birmingham, Birmingham, UK

${ }^{\mathrm{f}}$ Center for Collaboration and Innovation in Brain and Learning Sciences, Beijing Normal University, Beijing, China

* Corresponding Author:

Yan Song

State Key Laboratory of Cognitive Neuroscience and Learning

Beijing Normal University

Beijing 100875, China

Tel: 86-10-5880-4267

Fax: 86-10-5880-6154

Email: songyan@bnu.edu.cn

${ }^{1}$ These authors contributed equally to this work.

Running Title: tPBM improves visual working memory capacity 


\section{Abstract}

Transcranial photobiomodulation (tPBM) is a novel and noninvasive intervention, which has shown

t0 promise for improving cognitive performances. Whether TPBM can modulate brain activity and thereby enhance

H1 working memory (WM) capacity in humans remains unclear. In this study, we delivered double-blind and 12 sham-control tPBM with different wavelengths to the prefrontal cortex (PFC) in 90 healthy participants and t3 conducted four electroencephalography (EEG) experiments to investigate whether individual visual working 14 memory capacity and related neural response could be modulated. We found that $1064 \mathrm{~nm}$ tPBM applied to the 15 right PFC has both a substantial impact on visual working memory capacity and occipitoparietal contralateral t6 delay activity (CDA), no matter orientation or color feature of the memorized objects. Importantly, the CDA 17 set-size effect during the retention mediated the effect between $1064 \mathrm{~nm}$ tPBM and subsequent WM 18 performance. However, these behavioral benefits and the corresponding changes of CDA set-size effect were 19 absent with tPBM at $852 \mathrm{~nm}$ wavelength or with the stimulation on the left PFC. Our findings provide 50 converging evidence that $1064 \mathrm{~nm}$ tPBM applied on the right PFC can improve visual working memory ¡1 capacity, as well as explain the individual's electrophysiologyical changes about behavioral benefits.

Key words: transcranial photobiomodulation, EEG, event-related potentials, CDA, visual working memory 


\section{Introduction}

35 Working memory (WM) - the ability to actively store useful information 'in mind' in a matter of

seconds-plays a vital role in many cognitive functions. Individual differences in WM capacity predict fluid intelligence and broad cognitive function ${ }^{1}$, which has made increasing WM capacity become an attractive aim ;8 for interventions and enhancement. In the past decades, non-invasive brain stimulation (NIBS) technology ;9 involving the transcranial application of electrical (direct or alternating) or magnetic fields to the specific scalp 50 or multiple brain circuits, has been proven to be useful for improvement in WM performance. The NIBS 51 research has found that behavior enhancement was associated with linked neurophysiological changes, such as 52 increased functional connectivity between brain regions ${ }^{2}$ and oscillatory neuronal activity $^{3}$, as well as 33 event-related potentials ${ }^{4}$.

Recently, photobiomodulation (PBM) has been applied to modulate the metabolic processes in the brain, and it has emerged as a promising intervention to improve cognitive functions. It has been suggested that PBM up-regulates the complex IV of the mitochondrial respiratory chain to modulate cytochrome c oxidase (CCO). This leads to increased adenosine triphosphate (ATP) formation and initiate secondary cell-signaling

pathways $^{5-7}$. The resulting metabolic effects following PBM increases the cerebral metabolic energy production, oxygen consumption, and blood flow in animals and humans ${ }^{8-10}$. In addition, some studies suggest that PBM can enhance neuroprotection by modulating the neurotrophic factors and inflammatory signaling molecules as well as anti-apoptotic mediators ${ }^{11}$.

Transcranial photobiomodulation (tPBM) has been a noninvasive method of targeting the brain via wavelength between 620 and $1100 \mathrm{~nm}$. Rojas ${ }^{12}$ demonstrated that $660 \mathrm{~nm}$ tPBM could improve prefrontal cortex (PFC) oxygen consumption and metabolic energy, thereby increasing PFC-based memory functions in rats. Other studies have shown $1072 \mathrm{~nm}$ tPBM can reverse middle-aged mice's deficits in working memory ${ }^{13}$. These animal findings suggest that the oxygen metabolism of cortical tissue exposed to PBM is enhanced and that this can result in the enhancement of memory. Two human behavioral studies have shown that $1064 \mathrm{~nm}$ tPBM over the right PFC can improve accuracy and speed up reaction time in WM tasks ${ }^{14,15}$. Meanwhile, other 
79 behavioral studies suggested that some high-order cognitive functions could also be improved after tPBM 30 therapy, such as sustained attention and emotion ${ }^{14}$, as well as executive functions ${ }^{16}$.

However, the performance of even the simplest WM task involves multiple cognitive processes, such as

perceptual encoding, selective attention, and motor execution, which might confound the associations between

tPBM effect and WM enhancement. Taking this into account, we chose the K-value estimates to assess the accurate number of items maintained in the visual WM for the given load array ${ }^{17}$. Given that right PFC was associated with information maintenance in $\mathrm{WM}^{18}$, we hypothesized that $1064 \mathrm{~nm}$ tPBM over the right PFC (Fig. 1A) leads to behavioral enhancements in visual WM capacity. However, we still lack WM-related neurological evidence to directly bridge the gap between $\mathrm{TPBM}$ effects and WM behavioral benefits. Previous studies have extensively demonstrated that contralateral delay activity (CDA) tracks the number of objects stored in visual WM. Furthermore, the set-size effects of the CDA (defined as the increase in amplitudes from set-size two to set-size four) predicted the individual differences in WM capacity ${ }^{19}$. Thus, we linked behavioral benefits (K value) in WM capacity from tPBM with measurable and identified ERP biomarkers (CDA) of WM capacity.

We conducted four double-blind, sham-controlled tPBM experiments (Fig.1A), in which participants completed two different sessions of tPBM that were separated by a week between sessions, with sham or active tPBM on the PFC, respectively (Fig.1B). After stimulation, participants performed a classical change detection task in which WM load was manipulated (high versus low load, Fig.1C) while recording the electroencephalography (EEG). The classical change detection task requires participants to maintain the features of items (orientation for Experiment 1; color for Experiment 2) at the cued side in WM for subsequent recognition, which reliably induces sustained CDA componets. Then, we report the results from a series of follow-up experiments, which explore the specificity of tPBM in terms of wavelengths (Experiment 3) and stimulation sites (Experiment 4) for the enhancement of WM capacity and extend the principal behavioral and EEG of Experiment 1 and 2. 


\section{Results.}

\section{4nm tPBM on the right PFC enhanced individual WM capacity.}

55 Two classic change detection tasks were implemented to asesss WM performance, which require participants to )6 remember the orientations (Experiment 1) or color (Experiment 2) of a set of items in the cued hemifield (see

7) Fig. 1C). Individual WM capacity was assessed by calculating the K value according to hit and false alarm rates

)8 (see Materials and Methods) under the high-load and low-load conditions. A two-way mixed-effect ANOVA

)9 with tPBM stimulation (sham, active; within-subjects) and tasks (orientation, color; between subjects) as factors

$\left\lfloor\right.$ was conducted on behavioral $\mathrm{K}$ values. The results revealed a significant main effect of $\mathrm{tPBM}$ stimulation $\left(F_{1,40}\right.$

$\left\lfloor=13.436, p<0.001, \eta_{p}{ }^{2}=0.925\right)$ but no significant tPBM stimulation-by-task interaction $\left(F_{1,40}=0.080, p=\right.$

I2 $\left.0.779, \eta_{p}{ }^{2}=0.006\right)$. Specifically, compared with sham-tPBM, behavioral results showed that $\mathrm{K}$ values increased

13 after active-tPBM with $1064 \mathrm{~nm}$ both in the orientation WM task (Experiment 1: $t_{24}=2.841, p=0.009$, Cohen's

I4 $\mathrm{d}=0.568$, two-tailed) and in the color WM task (Experiment 2: $t_{17}=2.760, p=0.013$, Cohen's $\mathrm{d}=0.651$,

L5 two-tailed). The mean tPBM effect (Active minus Sham) on $\mathrm{K}$ value for Experiment 1 was $0.186 \pm 0.065\left(\mathrm{BF}_{10}\right.$

$16=5.212)$, and the mean tPBM effect for Experiment 2 was $0.188 \pm 0.051\left(\mathrm{BF}_{10}=20.336\right)$. This result supported

17 the hypothesis that $1064 \mathrm{~nm}$ tPBM on the right PFC can enhance individual WM capacity.

18 Several studies in neuro-enhancement, e.g., transcranial direct current stimulation (tDCS), showed a

19 performance-dependent stimulation effect with generally stronger effects only for the individual with low WM

20 capacity $^{4,20}$. To examine this effect with respect to tPBM, we divided participants into two sub-groups based on

21 their averaged $\mathrm{K}$ values in the orientation WM task under sham-tPBM stimulation in Experiment 1 ( $\mathrm{n}=13$ and

22 n=12 for low- and high-performance groups, respectively). A two-way mixed-effect ANOVA showed no

23 significant interaction between tPBM stimulation and sub-group $\left(F_{1,23}=1.170, p=0.291, \eta_{p}^{2}=0.110\right)$,

24 suggesting a lack of performance-dependent effect. That is, both good and poor WM capacity could be

25 improved after $1064 \mathrm{~nm}$ tPBM. A similar analysis involving the color WM task also showed no

26 performance-dependent effects in Experiment $2\left(F_{1,15}=0.002, p=0.963, \eta_{p}^{2}<0.001\right)$. 
27 Our results in Experiments 1 and 2 demonstrate that participants could maintain more items in visual WM

28 with external $1064 \mathrm{~nm}$ tPBM stimulation on the right PFC. These effect were independent of performance and

29 task. Importantly, participants could not report or guess whether they were assigned to sham or active tPBM.

30 Subjects guessed at chance level (see Fig.1B, hit rate $=48.1 \%$ ), suggesting they had no awareness of the tPBM. 


\section{A tPBM Protocol}

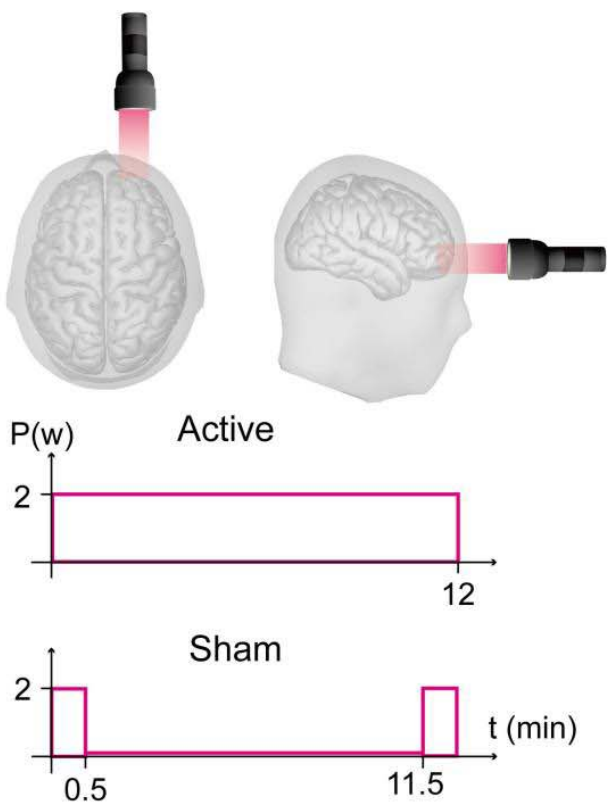

\section{Working Memory Tasks}
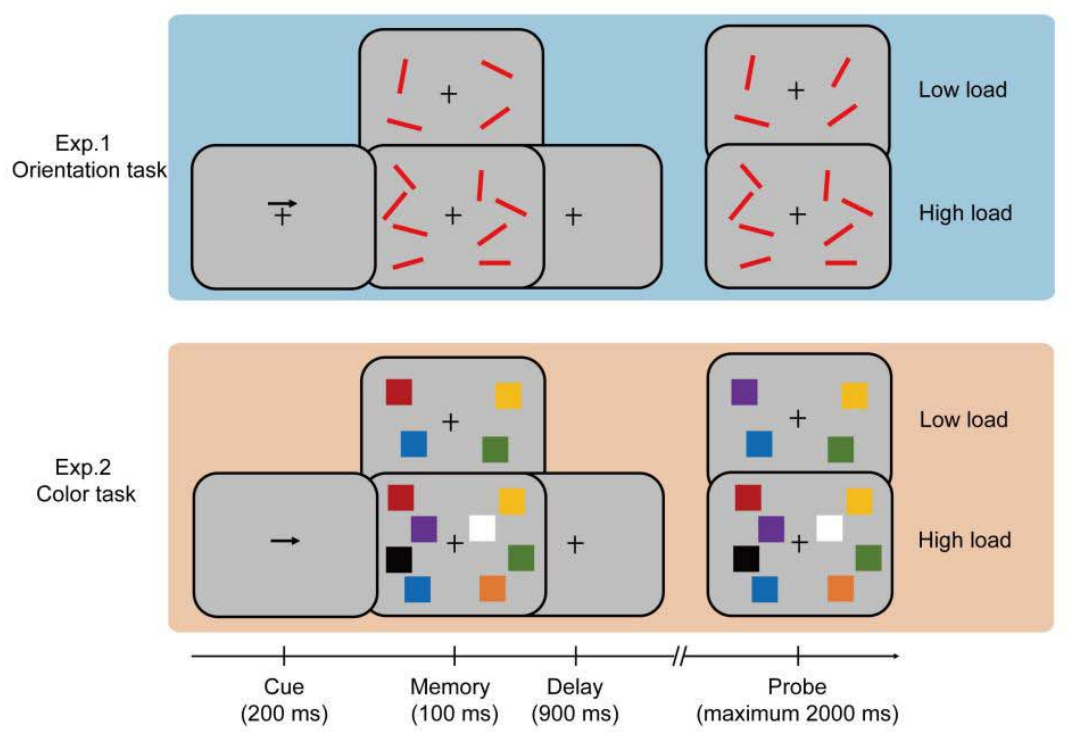

\section{B Experimental Protocol}

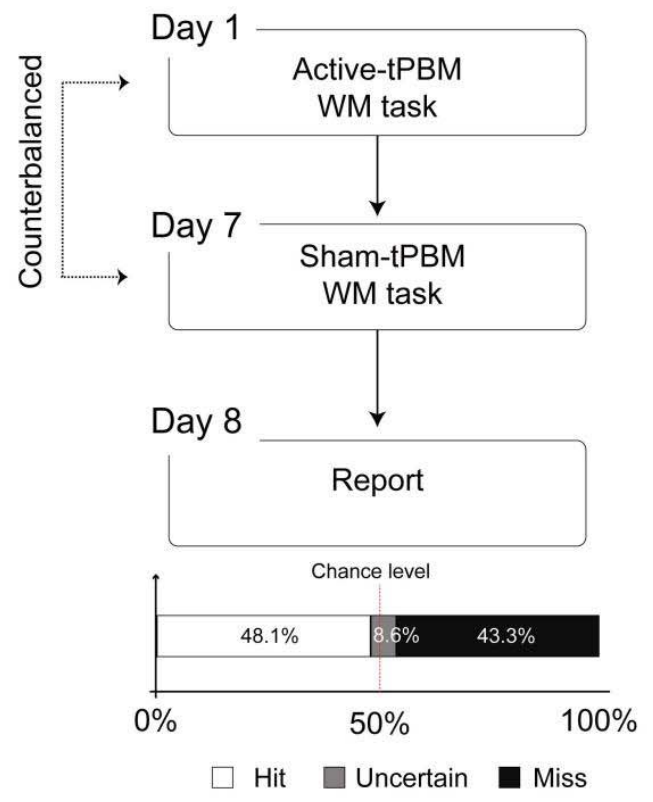

D Behavioral Results
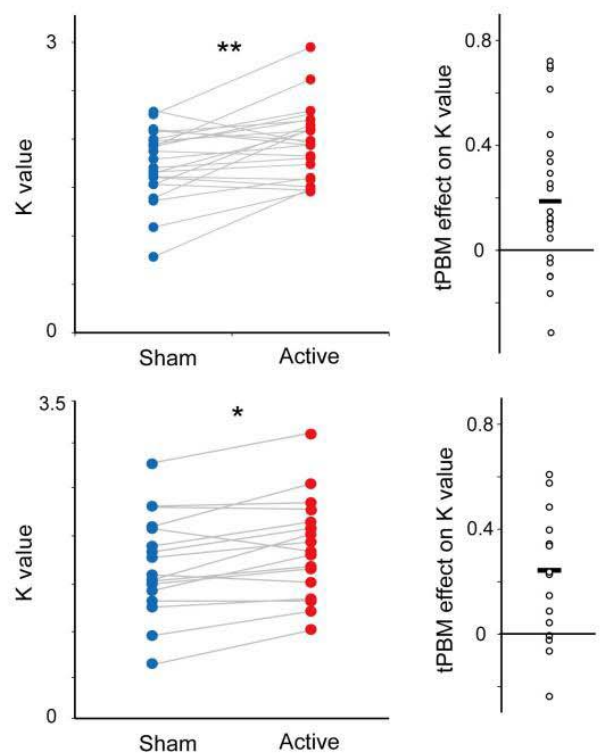

Figure 1. Protocol, task, and behavioral result in Experiment 1 and 2. (A) tPBM protocol. Active-tPBM was delivered by the laser with $1064 \mathrm{~nm}$ wave-length at the right PFC for a total of 12 -minute treatments. (B) Experimental protocol. Each participant received two tPBM sessions (active and sham, randomized, double-blind design), separated by one week. On the eighth day, participants were required to report or guess which session was active or sham tPBM. (C) WM tasks. In Experiment 1, the participants were required to perform an orientation WM task. In Experiment 2, the participant was required to perform a color WM task. Two tasks used the same relative timing and protocol, and the only difference between the two tasks is the memory dimension (orientation in Experiment 1; color in Experiment 2). Each participant only took part in one Experiment. (D) Left, performance in terms of $\mathrm{K}$ value for orientation WM task (up) and color WM task (down) under sham-tPBM (blue circles) and active-tPBM (red circles). Right, the tPBM effect on $\mathrm{K}$ value (active minus sham), The dots indicate individual performance. tPBM, transcranial photobiomodulation; PFC, prefrontal cortex; WM, working memory. ${ }^{*} p<0.05 ;{ }^{* *} p<0.01$. 
15

\section{CDA tracks the enhancement in individual WM capacity.}

The participants' EEG signals were simultaneously recorded while they performed the WM tasks. Consistent with previous studies ${ }^{19}$, the ERP results show a negative deflection at contralateral relative to ipsilateral scalp sites at PO7 and PO8 (see Supplementary). We defined the CDA amplitude set-size effect as the CDA amplitude of 2 objects (low-load) minus the amplitude of 4 objects (high-load). Note that we did not track the "raw" CDA amplitude, but rather the increase in CDA amplitude from low- to high-load as a dependent variable. To investigate the effect of tPBM on CDA amplitude set-size effect, a two-way mixed ANOVA on CDA amplitude set-size effect was conducted considering the WM task (orientation, color) and tPBM stimulation (sham, active) as factors. As expected, the results showed a significant main effect of tPBM stimulation $\left(F_{1,41}=\right.$ $\left.12.249, p=0.001, \eta_{p}{ }^{2}=0.227\right)$. The main effect of task $\left(F_{1,41}=0.660, p=0.421, \eta_{p}{ }^{2}=0.012\right)$ and the tPBM stimulation $\times$ task interaction $\left(F_{1,41}=0.474, p=0.495, \eta_{p}{ }^{2}=0.011\right)$ did not reach significance. Follow-up t-tests indicated that the CDA amplitude set-size effect during the delay period was significantly stronger in the active-tPBM session relative to the sham-tPBM session in both the orientation WM task $\left(t_{24}=2.313, p=0.030\right.$, Cohen's $d=0.463$, two-tailed) and color WM task ( $t_{17}=2.506, p=0.023$, Cohen's $d=0.591$, two-tailed $)$. The sLORETA source estimates (see Materials and Methods) of the CDA set-size effect are shown in Fig. 2. These results suggested that the significantly increased CDA amplitude set-size effects (active minus sham) were localized in the superior IPS for two WM tasks with $1064 \mathrm{~nm}$ tPBM applied over the right PFC (ps $<0.05)$.

To better understand the tPBM effect, we showed the $\mathrm{K}$ values and CDA set-size effects from different stimulation sessions across the orientation and color WM tasks (Fig. 2C). Although the K value and CDA set-size effect were evaluated as separate dimensions involving WM, as can be seen here, the changes in $\mathrm{K}$ values and CDA across the two tasks have similar trends induced by active-tPBM, relative to the sham-tPBM. This result is consistent with the hypothesis that significant behavior enhancements and CDA co-benefits are associated with the active-tPBM effect. Also as expected, the CDA set-size effect from the two experiments shows a task-independent tPBM effect. 


\section{Orientation task}
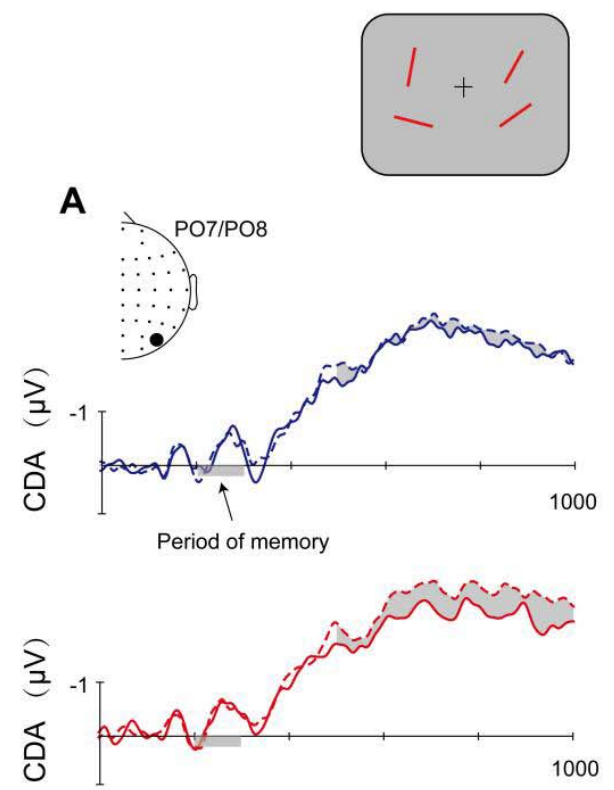

Time (ms)

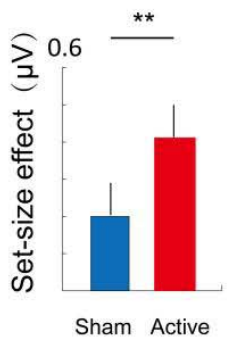

Active vs. Sham

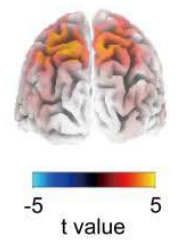

High load
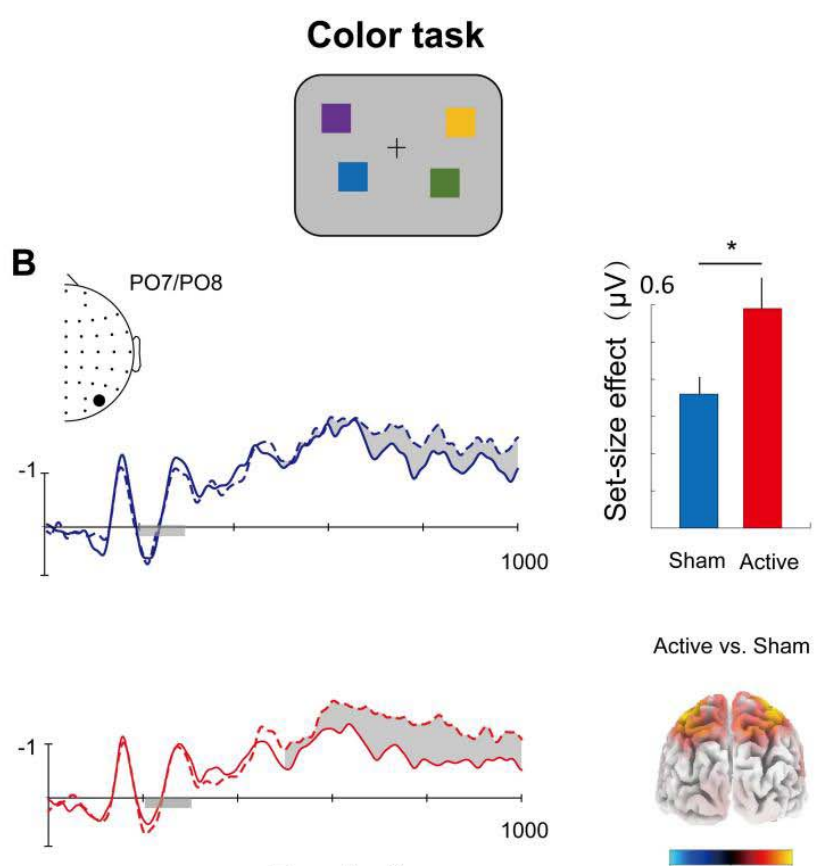

Time (ms)

Low load
Active vs. Sham

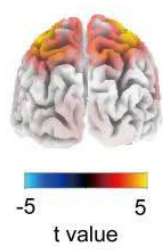

C tPBM effects (1064 nm, Right PFC)

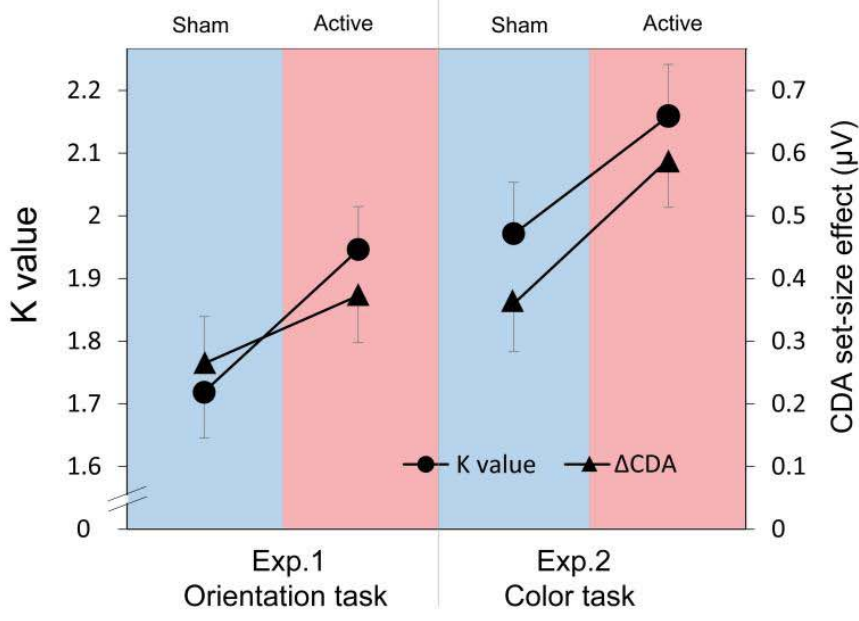

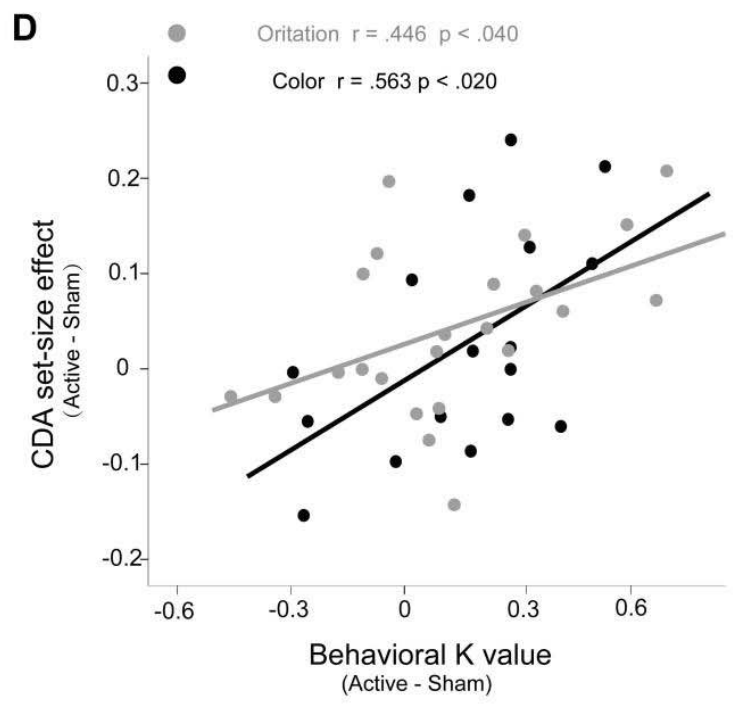

71 Figure 2. Grand average of event-related potentials (ERPs) for the orientation WM task in Experiment 1 (A) and color WM task in 12 Experiment 2 (B). Shading indicates the contralateral delayed activity (CDA) set-size effect. The enlarged black dots on EEG topographies show PO7/PO8 electrodes. Bar plots represent the average CDA set-size effect. Errors bars represent SEM. Significant set-size effects are located in the intraparietal sulcus (IPS). 3D brain map (t-map) backview of significant tPBM effect on CDA. (C) tPBM-effect. K value and CDA set-size effect for the two tasks (orientation WM task, color WM task) and two sessions (active-tPBM, sham-tPBM). Solid lines indicate the value is compared with-subject. (D) Scatterplots of participants' behavioral benefits (active minus sham) against the changes of CDA set-size effect (active minus sham) for the orientation WM task (gray) and the color WM task (black). tPBM, transcranial photobiomodulation; WM, working memory. ${ }^{*} p<0.05{ }^{* *} p<0.01$. 
Next, we were interested in testing whether the use of EEG recording could provide electrophysiology-linked

evidence of the beneficial tPBM effect. We performed Pearson correlation analyses at the subject level to

provide more detailed information on the relationships between CDA and behavior. As shown in Fig. 2D,

participants with stronger CDA amplitude set-size effect showed higher tPBM effect on the behavioral $\mathrm{K}$ value

(for orientation task: $r=0.446, p<0.040$; for color WM task: $r=0.563, p<0.020$ ). The results suggest, for

both color and orientation WM task, that the CDA amplitude set-size effects might be able to predict the

behavioral working-memory benefits from tPBM.

CDA mediates the working memory improvements with $\mathrm{PBM}(1064 \mathrm{~nm})$ applied to right PFC

Given the above significant relationship between the increase in CDA set-size effect and the increase in $\mathrm{K}$ value

after $1064 \mathrm{~nm}$ tPBM, relative to sham sessions. We performed a mediation analysis (see Materials and Methods)

to examine whether the effect of tPBM on WM capacity (reflected by $\mathrm{K}$ value) was mediated by the CDA set-size effect. Therefore, we considered the tPBM sessions (active vs. sham) as predictors, WM performance (K value) as the predicted variable, and CDA set-size effect as a mediator (see Fig. 3A). This mediation analysis revealed a significant indirect effect of CDA set-size effect (indirect effect: $0.300,95 \%$ confidence interval: -0.107 to $0.706, p=0.146$; direct effect: $0.245,95 \%$ confidence interval: 0.021 to $0.844, p=0.040$ ). Mediation analysis demonstrates the indirect effect of $1064 \mathrm{~nm}$-tPBM on behavioral $\mathrm{K}$ value through an increase in the amount of information maintained in visual WM, as reflected by increases of CDA set-size effect. We further correlated CDA set-size effect with behavioral $\mathrm{K}$ value across all sessions in Experiment 1 and Experiment 2. Correlational analysis showed that the change of CDA set-size effect strongly correlated with the change of behavioral $\mathrm{K}$ value ( $r=0.404, p<0.001$, confidence interval: 0.154 to 0.654 ; Fig. $3 \mathrm{~B}$ ), highlighting the robust, inherent relationship between CDA set-size effect and behavioral value. This result is consistent with previous research ${ }^{21}$ that CDA is indicative of the number of maintained objects in visual working memory. 

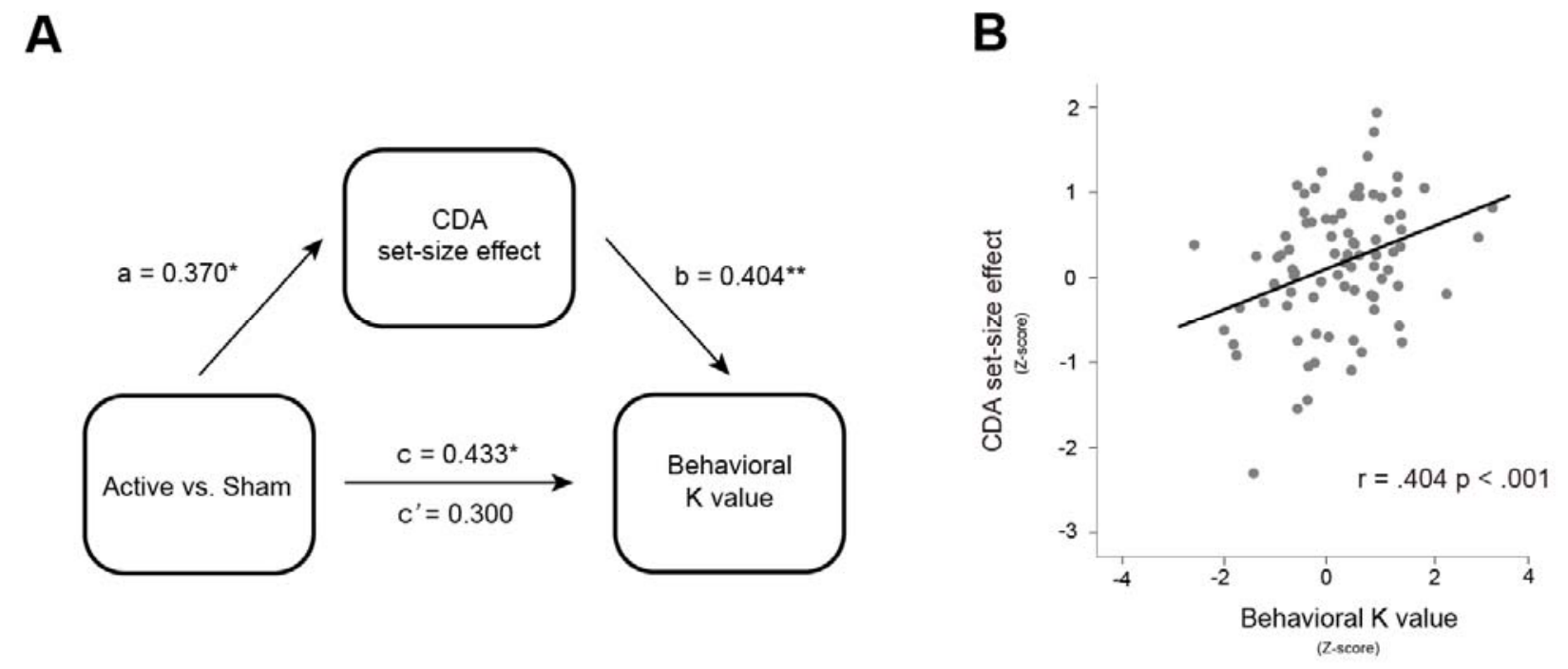

)4 Figure 3. CDA set-size effect mediated behavioral K values by 1064 nm-tPBM. (A) Mediation model demonstrating effect of

)5 $1064 \mathrm{~nm}$-tPBM on improved K values via increases in CDA set-size effect. a, b and c denote standardized beta coefficients of the )6 direct path strength. c' denotes the beta coefficient of path strength after controlling for changes of CDA set-size effect (B)

)7 Scatterplots of behavioral K value (Z-score) and the CDA set-size effect (Z-sore) across all participants (active- and sham- session) )8 in Experiment 1 and Experiment 2. tPBM, transcranial photobiomodulation; CDA, contralateral delayed activity, * $p<0.05, * * p$ ) $9<0.01$

The wave-length specificity of $\mathrm{PBM}$ on the enhancement of WM capacity.

12 We next considered whether exogenous heat from active-tPBM might alter the neural activity or influence 13 behavior relative to sham-tPBM sessions. In Experiment 3, we sought to disambiguate the effects of 4 photobiomodulation and tissue heating and examine the optical specificity of tPBM by using a laser light of 15 different frequency over the right PFC (852 nm, see Materials and Methods; Fig. 4A). We hypothesized that if

L6 heating could enhance individual WM capacity, we should expect to find the same enhancement in K value and 17 CDA amplitudes set-size effect after 852-tPBM as 1064-tPBM. Therefore, we use the same power and I8 stimulation duration as 1064-tPBM for 852-tPBM to control they produced the same quantity of heat. First, we 19 found that 852-tPBM did not modify the participants' behavioral $\mathrm{K}$ value for the orientation WM task, as 20 compared to sham-tPBM $\left(t_{19}=0.381, p=0.707\right.$, Cohen's $d=0.085$, two-tailed, Fig. 4B). The mean tPBM 21 effect (active minus sham) on $\mathrm{K}$ value for Experiment 3 was $-0.029 \pm 0.088\left(\mathrm{BF}_{10}=0.244\right)$. We compared the 22 data between $852 \mathrm{~nm}$ tPBM in Experiment 3 and $1064 \mathrm{~nm}$ tPBM in Experiment 1 (Fig. 4D). A two-way 
23 mixed-effect ANOVA on $\mathrm{K}$ values further revealed a significant tPBM stimulation (sham, active) and

24 wavelength $(1064 \mathrm{~nm}, 852 \mathrm{~nm})$ interaction $\left(F_{1,41}=4.474, p=0.041, \eta_{p}{ }^{2}=0.095\right)$, indicating that WM

25 performance improved significantly only in active-tPBM sessions applied $1064 \mathrm{~nm}$ wavelength, but not $852 \mathrm{~nm}$

26 wavelength. Similarly, 852-tPBM did not modulate CDA amplitude set-size in comparison to sham-tPBM for

27 orientation WM task ( $t_{19}=0.129, p=0.899$, Cohen's $d=0.030$, two-tailed, Fig. 4C). A two-way mixed-model

28 ANOVA on CDA amplitudes further revealed marginally significant tPBM stimulation (sham, active) and

29 wavelength $(1064 \mathrm{~nm}, 852 \mathrm{~nm})$ interaction $\left(F_{1,41}=3.623, p=0.064, \eta_{p}{ }^{2}=0.080\right)$. These results suggest that the

30 tPBM effect on WM is specific to the $1064 \mathrm{~nm}$ wavelength and heating does not play a role in the behavioral

31 and electrophysiological changes observed here. Subjects also guessed at chance level (hit rate $=47.8 \%$ ),

32 suggesting they had no awareness of the $852 \mathrm{~nm}$ tPBM over the right PFC.

\section{A Stimulation Protocol (852 nm vs. $1064 \mathrm{~nm})$}
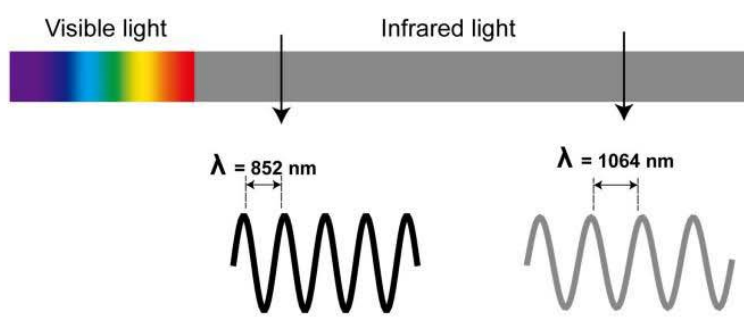

C EEG (852 $\mathrm{nm}$, orientation task)
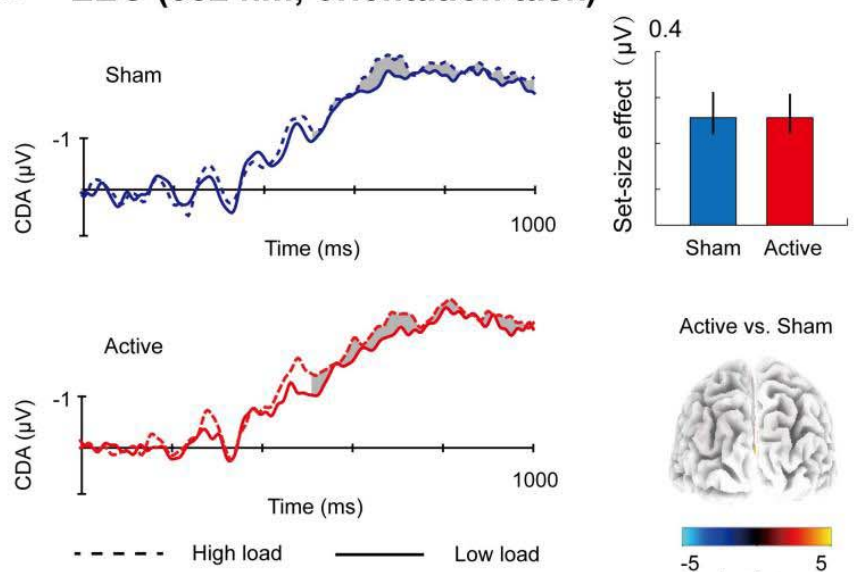

34

\section{B Behavioral Result (852 $\mathrm{nm}$, orientation task)}

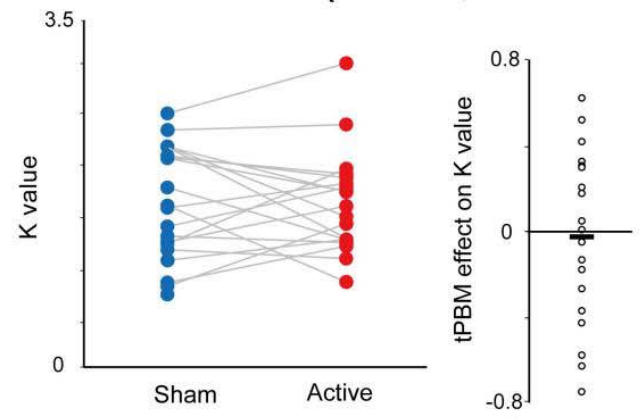

D tPBM effect (Right PFC, oritention task)

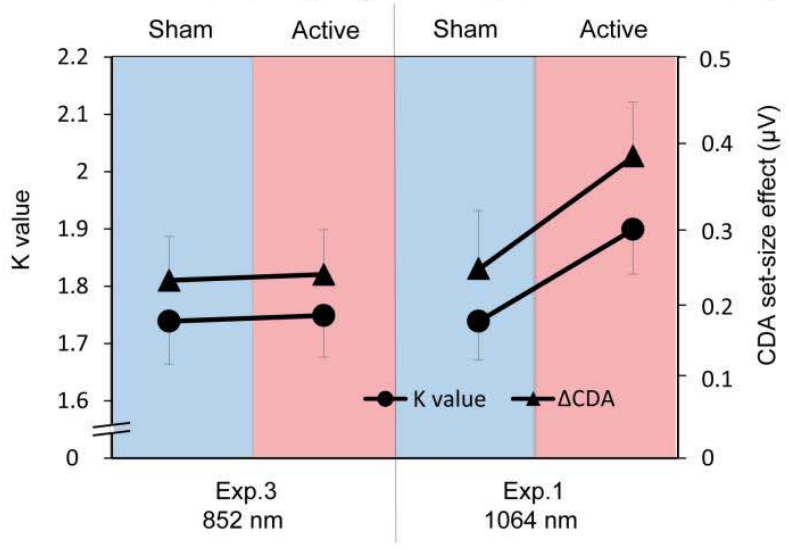

Figure 4. (A) Stimulation protocol. Active-tPBM was delivered by the laser light with $852 \mathrm{~nm}$ wave-length over right PFC in Experiment 3 (black sine wave) or $1064 \mathrm{~nm}$ wave-length in Experiment 1, 2 and 4 (gray sine wave). (B) In terms of $\mathrm{K}$ value for tPBM stimulation (sham, active) for orientation WM task in Experiment 3. The circles indicate individual performance. (C) Grand 
average of event-related potentials for active $852 \mathrm{~nm}$ - and sham-tPBM session. Shading indicates the CDA set-size effect. Bar plots represent the average CDA set-size effect: blue, sham session, red, active session. Errors bars represent SEM. 3D brain map (t-map) backview of significant tPBM effect on CDA. (D) $\mathrm{K}$ value and CDA set-size effect for the orientation WM task in Experiment 3 (852nm tPBM) and Experiment 1 (1064 nm tPBM). tPBM, transcranial photobiomodulation; PFC, prefrontal cortex; WM, working memory.

1064nm tPBM on the left PFC could not enhance individual WM capacity.

15 In Experiment 4, we use the same power, stimulation duration, but left PFC as a control stimulation location to t6 examine whether the $1064 \mathrm{~nm}$ tPBM could enhance the WM capacity regardless of the location of stimulation (Fig. 5A). The task in Experiment 4 is the same orientation WM task as Experiment 1. Figure 4 showed that 18 compared to sham-tPBM, 1064nm tPBM on left PFC did not enhance the individual behavioral $\mathrm{K}$ value $\left(t_{19}=0\right.$. $19381, p=0.707$, Cohen's $d=0.085$, two-tailed, Fig. 5B) and corresponding CDA set-size effect $\left(t_{19}=0.129, p=\right.$ ;0 0.899, Cohen's $d=0.030$, two-tailed, Fig. 5C). The mean tPBM effect (active minus sham) on $\mathrm{K}_{\max }$ value for ;1 Experiment 4 was $-0.032 \pm 0.058\left(\mathrm{BF}_{10}=0.258\right)$. We compared the data between tPBM applied on the left PFC ;2 in Experiment 4 and tPBM applied on the right PFC in Experiment 1 (Fig. 5D). A two-way mixed-model ;3 ANOVA further revealed significant interactions between tPBM stimulation (sham, active) and location (left, ;4 right $)$ on both $\mathrm{K}$ values $\left(F_{1,42}=4.474, p=0.041, \eta_{p}{ }^{2}=0.095\right)$ and on CDA set-size effect $\left(F_{1,42}=2.623, p=\right.$ ;5 $\left.0.044, \eta_{p}{ }^{2}=0.098\right)$, indicating that WM performance improved significantly only in $1064 \mathrm{~nm}$ tPBM sessions ;6 applied on right PFC, but not on left PFC. Subjects also guessed at chance level (hit rate $=46.9 \%$ ), suggesting ;7 they had no awareness of the tPBM over left PFC. 


\section{A Stimulation Protocol (Left PFC)}

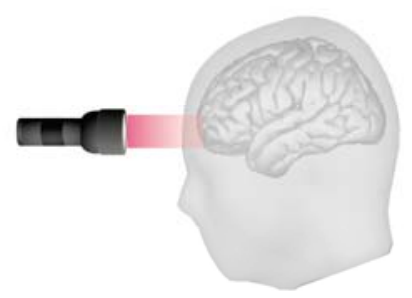

\section{EEG (Left, orientation task)}
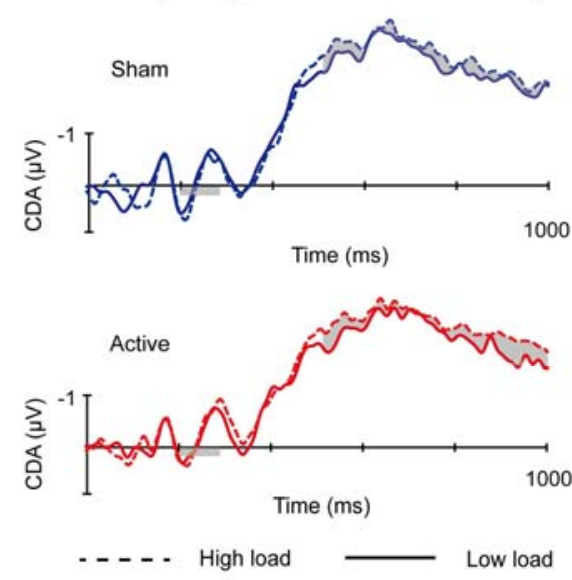

\section{B Behavioral Result (Left, orientation task)}

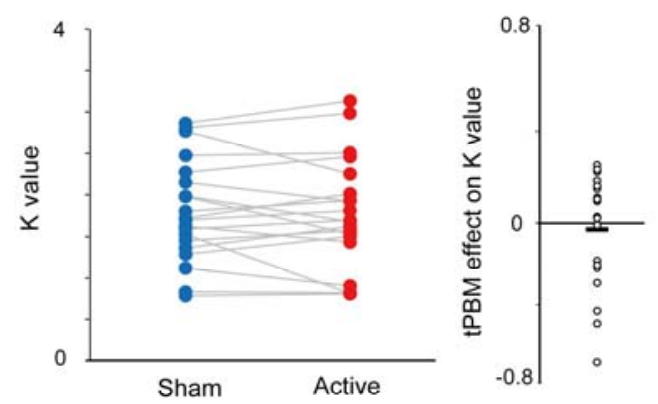

D tPBM effect (1064 $\mathrm{nm}$, orientation task)
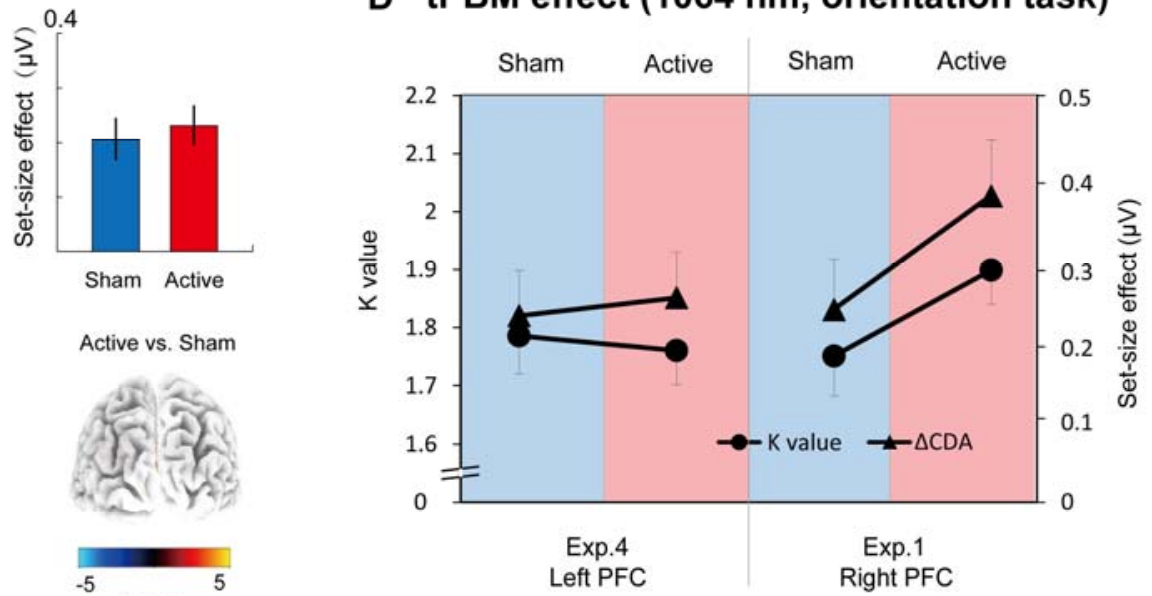

Figure 5. (A) Stimulation Protocol of Experiment 4. Active-tPBM was delivered by the laser with $1064 \mathrm{~nm}$ wave-length at the left prefrontal cortex for a total of 12 -minute treatments. (B) In terms of K value for tPBM stimulation (active, sham) applied on left PFC in Experiment 4. Each circle indicates individual performance. (C) Grand average of event-related potentials for $1064 \mathrm{~nm}-$ and sham-tPBM session in Experiment 4. Shading indicates the CDA set-size effect. Bar plots represent the average CDA set-size effect: blue, Sham session, red, Active session. Errors bars represent SEM, 3D brain map (t-map) backview of significant tPBM effect on CDA. (D) $\mathrm{K}$ value and CDA set-size effect for the orientation WM task in Experiment 4 (tPBM stimulation applied on the left PFC) and in Experiment 1 (tPBM stimulation applied on the right PFC). tPBM, transcranial photobiomodulation; PFC, prefrontal cortex; WM, working memory. 


\section{Time course of the enhancement in WM after $\mathrm{TPBM}$.}

70 To investigate the emergence of the behavioral enhancement across blocks, we calculated the $\mathrm{K}$ values of

71 high-load conditions across the four blocks in all four experiments (Fig. 6). Paired t-test showed, relative to

12 sham session, significant behavioral enhancements were only found during the late period in Experiment 1

13 (block 3: $t_{24}=3.840, p<0.001$, Cohen's $d=0.768$, two-tailed; block 4: $t_{24}=2.155, p=0.041$, Cohen's $d=0.504$,

14 two-tailed) and Experiment 2 (block 3: $t_{17}=2.137, p=0.047$, Cohen's $d=0.431$, two-tailed) with $1064 \mathrm{~nm}$

$75 \mathrm{tPBM}$ on right PFC, but not found in other blocks or experiments $(p s>0.050)$. We further compared $\mathrm{K}$ value in

16 block 3 across Experiment 1 and Experiment 3 when applying tPBM over the right PFC, 1064 nm stimulation

77 relative to $852 \mathrm{~nm}$ enhanced the $\mathrm{K}$ values of working-memory behavior $\left(t_{42}=2.795, p=0.008\right.$, Cohen's $d$

$78=0.838$, two-tailed). When applying tPBM with the same $1064 \mathrm{~nm}$ wavelength, stimulation over the right PFC

19 relative to the left PFC enhanced the K values of working-memory behavior $\left(t_{43}=1.959, p=0.056\right.$, Cohen's $d$

$30=0.580$, two-tailed). No significant differences were found in other blocks ( $p s>0.050)$. 


\section{A Orientation task}

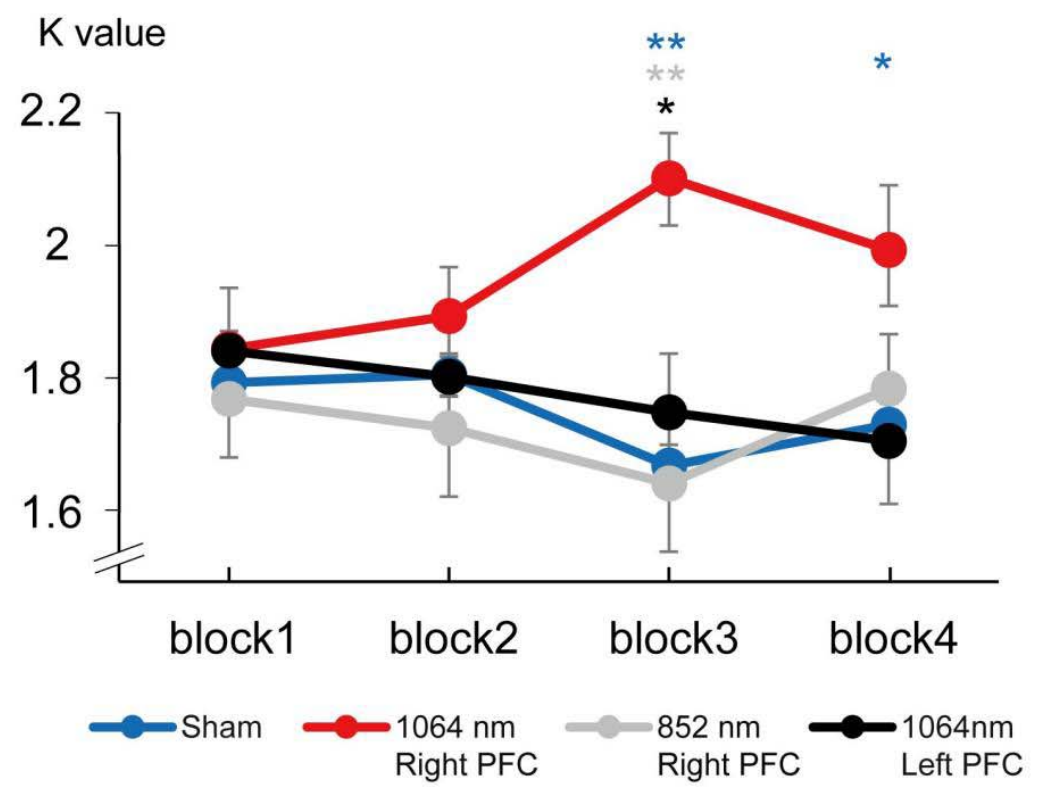

\section{B Color task}

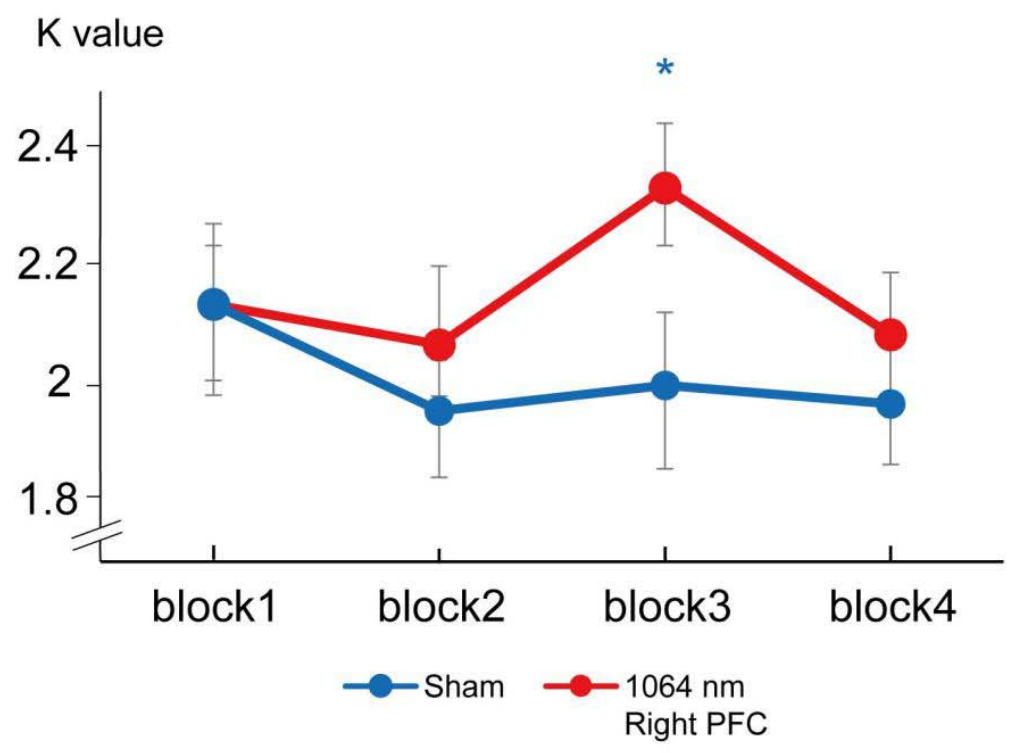

33 Figure 6. (A) K values across the four blocks in the orientation WM task in Experiments 1, 3 and 4. Blue line represents the sham

34 session in Experiment 1. (B) $\mathrm{K}$ values across the four blocks in the color WM task in Experiment 2. Blue line represents the sham 35 session in Experiment 2. WM, working memory. ${ }^{*} p<0.05 ;{ }^{* *} p<0.01$. 
No evidence for the contralateral enhancement or ipsilateral inhibition after 1 PBM.

Our results showed that the increase in CDA set-size effects track WM capacity benefits after $1064 \mathrm{~nm}$ tPBM

applying on the right PFC. Previous studies proposed that CDA reflected the inhibition of ipsilateral

waves of memory objects, it is necessary to examine tPBM effect on both contralateral and ipsilateral ERPs (Supplementary Figures $1-4$ ). However, we did not find a robust difference between active and sham tPBM for contralateral or ipsilateral ERPs, suggesting that the tPBM effect might not simply contribute to only the contralateral or ipsilateral hemifield of memory objects. Further work is needed to explore the tPBM effect on task-relevant contralateral enhancement and task-irrelevant ipsilateral inhibition during WM retention.

\section{Materials and Methods.}

\section{Participants.}

Neurologically normal college students $(\mathrm{n}=90)$ with normal or corrected-to-normal vision took part in four experiments. 27 of whom participated in experiment $1(5$ male, mean age $=22)$. No statistical methods were used to pre-determine the sample size, but the sample size was chosen to be adequate to receive significant results as determined by preliminary experiments. Because the identified tPBM effect on CDA amplitudes in experiment 1 was robust, we set the sample size to 21 in experiments $2-4$ (experiment 2: 7 male, age range $=$ $22.753 \pm 3.750$; experiment $3: 8$ males, age range $=22.655 \pm 4.050 ;$ experiment $4: 7$ male, age range $=22.808 \pm$ 3.955). For the EEG analysis, data from 12 participants (four in experiment 1 , three in experiment 2, three in experiment 3, two in experiment 4) were excluded due to incomplete data or low EEG quality. The Institutional Review Board approved the experimental procedures of Beijing Normal University, and informed consent was obtained from each participant.

\section{Experimental Protocol.}

Each participant only took part in one of four experiments. Each experiment consisted of one active tPBM session and one sham tPBM session completed on the first and seventh days. The order of two sessions was 
counterbalanced across participants (see Fig. 1B). On the eighth day, participants were required to report (or

guess) which session was the active tPBM session. Before EEG recordings, all subjects participated in a training

block to ensure that they could perform the tasks above chance level and check for potential EEG artifacts.

\section{tPBM Protocol}

The measured uniform laser beam has an area of $13.57 \mathrm{~cm}^{2}$ (4 centimeters diameter) and a continuous power output of 2271 milliwatts $(\mathrm{mW})$, resulting in an irradiance or power density of 167 milliwatts $/ \mathrm{cm}^{2}$ (2271 $\mathrm{mW} / 13.57 \mathrm{~cm}^{2}=167 \mathrm{~mW} / \mathrm{cm}^{2}$ ). At this power level, the energy emitted by the laser is one-fifth of the skin's maximum permissible exposure $\left(167 \mathrm{~mW} / \mathrm{cm}^{2}\right)$, exposure to it is not deemed harmful to tissue, and it causes no detectable physical damage and imperceptible heat. We performed a handheld stimulation on human tissue. The stimulation site in our experiment was centered on the FP2 electrodes (Experiments 1,2 and 3) or the FP1 electrode (Experiment 4) based on the 10-20 system used for EEG electrode placement (Fig.1A, upper panel). Each subject will be instructed to sit on a chair, adjusted to ensure comfort throughout the measurement. The room's ambient lighting will be shut down to ensure that it does not contaminate the laser light. Participants are instructed to wear protective eyewear and keep their eyes closed, as required by the laser manufacturer and the Beijing Normal University Laser Safety Program. In the active-tPBM session, the area stimulated (a 60 s/cycle, total laser energy per cycle $=2.271 \mathrm{~W} \times 60 \mathrm{~s}=136.26 \mathrm{~J} / \mathrm{cycle}$ ) alternated between sites medial and lateral to the right forehead (FP2) for 12 minutes before EEG recording. The sham-tPBM session received two brief 0.5-minute treatments (the first one at the beginning of the stimulation and the second in the end) to the intended site on the forehead, separated by 11-minutes of no treatment (laser power will be tuned down to $0 \mathrm{~W}$; Fig. 1A, lower panel). Thus, the sham-tPBM session received approximately 1/12th of the cumulative energy density as the active session. This 0.5 -minute treatment was a necessary part of the active placebo session by providing a similar subjective experience to the active-tPBM session. Since slight and brief laser light does not produce physiological or cognitive effects ${ }^{14,23}$. For tPBM stimulation, $1064 \mathrm{~nm}$ was used for Experiment 1,2 and 4; $852 \mathrm{~nm}$ was used for Experiment 3. The two nanometers were controlled to release equal optical energy. 
37 Each session will last 45-60 min (10-12 min tPBM stimulation, 8 min rest, and 25-30 min EEG recording). The

38 active and sham sessions will be divided at least one week to avoid the tPBM overlapping effects.

\section{t0 Working Memory Task.}

H1 The stimuli were presented on a 21 -inch liquid crystal display monitor $(1200 \times 768$ pixels, $120 \mathrm{~Hz}$ refresh rate $)$ H2 with a homogeneous light gray background $\left(12 \mathrm{~cd} / \mathrm{m}^{2}\right.$, RGB: $\left.125,125,125\right)$ at a distance of $65 \mathrm{~cm}$. At the H3 beginning of each trial, a $200 \mathrm{~ms}$ central arrow cue instructed the participants to remember the left or the right 14 hemifield objects. Next, the memory array was presented for $100 \mathrm{~ms}$, followed by a $900 \mathrm{~ms}$ interval. Then, the t5 probe array was presented for a maximum of $2000 \mathrm{~ms}$ or until response. Participants were instructed to respond t6 as quickly and accurately as possible whether the orientation or color of objects in the cue-side has changed 17 after a working memory delay.

18 In the orientation WM task, all memory arrays were presented within two $4^{\circ} \times 7.3^{\circ}$ rectangular regions that 19 were centered $3^{\circ}$ to the left and right of a black central fixation cross $\left(0.5 \mathrm{~cd} / \mathrm{m}^{2}, 0.4^{\circ} \times 0.4^{\circ}\right)$. Each memory array j0 consisted of two or four red oriented bars ( $2^{\circ}$ in length and $0.5^{\circ}$ in width) in each hemifield selected randomly ¡1 between $0^{\circ}$ to $180^{\circ}$, with the constraint that the orientations among bars within a hemifield were at least $20^{\circ}$ difference. Bars' positions were randomized on each trial, with the constraint that the distance between bars

within a hemifield was at least $2^{\circ}$ (center to center). The orientation of one bar in the probe array was different from the corresponding object in the memory array in 50\% of trials in each hemifield; the two arrays' orientations were identical on the remaining trials. In the color WM task, each memory array consisted of two or four colored squares $\left(1^{\circ} \times 1^{\circ}\right)$ in each hemifield. Each square was selected randomly from a set of nine colors (red, green, blue, yellow, violet, pink, orange, black, and white). In the low-load condition, one square was presented in each quadrant. In the high-load condition, two squares were presented in each quadrant. In the probe array, the color of one square was different from the corresponding object in the memory array in $50 \%$ of trials in each hemifield; the two arrays' colors were identical on the remaining trials. Each session involved 8 blocks (4 low-load blocks and 4 high-load blocks, randomized across blocks). Each block contained 60 trials, and an 1-minute break separated adjacent blocks. In total, we collected 960 trials within about 30 minutes in each 
experiment per participant. Experiment 3 and 4 were identical to the same orientation WM task in Experiment 1, except that participants were assigned to active-tPBM with different wave-lengths (852 nm) in Experiment 3 and on other site (left PFC) in Experiment 4.

We computed visual memory capacity with a standard formula ${ }^{19}$ that essentially assumes that if an observer can hold in memory K items from an array of $\mathrm{S}$ items, then the item that changed should be one of the items being held in memory on K/S trials, leading to correct performance on K/S of the trials on which an item changed. The formula is $\mathrm{K}=\mathrm{S} \times(\mathrm{H}-\mathrm{F})$, where $\mathrm{K}$ is the memory capacity, $\mathrm{S}$ is the load of the array, $\mathrm{H}$ is the observed hit rate, and $\mathrm{F}$ is the false alarm rate. We evaluated the WM capacity according to $\mathrm{K}$ value under the high-load condition.

\section{EEG recording and analysis.}

The participants' EEG signals were simultaneously recorded while they performed the tasks. The EEG data were acquired using a SynAmps EEG amplifier and the Curry 8.0 package (NeuroScan, Inc.) from a Quick-cap with 64 silver chloride electrodes arranged according to the international 10-20 system. To detect eye movements and blinks, vertical eye movements were recorded from two vertical electrooculogram electrodes placed $1 \mathrm{~cm}$ above and below the left eye, while horizontal eye movements were recorded from two horizontal electrooculogram electrodes placed at the outer canthus of each eye. All electrodes, except those for monitoring eye movements, were referenced online to the left mastoid. Electrode impedance was kept below $5 \mathrm{k} \Omega$. The EEG was amplified at $0.01-200 \mathrm{~Hz}$ and digitized online at a sampling rate of $500 \mathrm{~Hz}$.

The data were processed in MATLAB (The MathWorks Inc., Natick, MA) using the ERPLAB toolbox and custom codes. They were preprocessing involved applying a $0.01-40 \mathrm{~Hz}$ bandpass filter and re-referencing data offline to the average of all electrodes. The EEG data were then segmented relative to memory array onset (from -200 to $900 \mathrm{~ms}$ ). Independence component analysis (ICA) was performed to correct eye-blink artifacts by semiautomatic routines for the segmented data. Epochs were automatically excluded from averaging if the EEG exceeded $\pm 100 \mu \mathrm{V}$ at any electrode or if the horizontal EOG exceeded $\pm 30 \mu \mathrm{V}$ from 0 to $500 \mathrm{~ms}$ around cue array onset. Then, epochs that continued to show artifacts after this process were subsequently detected and 
removed by the eye. Data from nine participants (three in experiment 1 , two in experiment 2 , two in experiment

3 , two in experiment 4 ) were discarded because of the high ratio of excluded trials ( $>40 \%$ of trials). Among the

participants' final set, artifacts led to the rejection of an average of $12.3 \%$ of trials per participant (range

$0.4-27.6 \%)$

For ERP processing, we focused on the ERP triggered by the memory array. The baseline correction was calculated for $200 \mathrm{~ms}$ before memory display onset in each trial. The trials were then averaged for each condition to create the ERP response. Contralateral waveforms were computed by averaging the right electrode sites for trials on which to-be-remembered objects occurred on the left side with the left electrode sites for trials on which to-be-remembered objects occurred on the right side. Ipsilateral waveforms were computed by averaging the right electrode sites for trials on which to-be-remembered objects occurred on the right side with the left electrode sites for trials on which to-be-remembered objects occurred on the left side.

The CDA was measured at the posterior parietal (PO7/PO8) as the difference in mean amplitude between the ipsilateral and contralateral waveforms, with a measurement window of 500-1000 ms after the onset of the cue array. In this study, the memory display could induce an N2pc before the CDA component ${ }^{24}$. To obtain a pure CDA measure without contamination of the N2pc component, we began the CDA measurement period at 500 ms, by which time the N2pc had ordinarily terminated. Note that the target's contralateral waveform was the average of the left-hemisphere electrodes when the target was in the right visual field and the right-hemisphere electrodes when the target was in the left visual field. Similarly, the ipsilateral waveform for the target was the average of the left-hemisphere electrodes when the target was in the left visual field and the right-hemisphere electrodes when the target was in the right visual field.

During ERPs analysis in the visual WM task, we detected the difference of CDA between groups in active and sham sessions with t-statistics analysis. For each comparison, a test was calculated for time-samples in ERP components with 5000 random permutations.

\section{Source locations}

The three-dimensional (3-D) distribution of the tPBM effect (Active minus Sham) in electrical activity was analyzed for each subject using the LORETA software ${ }^{25}$. Localization of the CDA set-size effect between the 
14 active session and the sham session was assessed by voxel-by-voxel paired t-tests of the LORETA images. To

15 correct for multiple comparisons, a nonparametric permutation test was applied $(\mathrm{p}<0.050$, determined by 5000

16 randomizations). Finally, the result values were shown with a 3-D brain model and evaluated for the level of

$17 \quad$ significances.

\section{$18 \quad$ Statistical analysis}

19 Mediation analysis for multilevel data was performed in the SPSS statistics package (version 20.0). three 20 models were built in the analysis: (1) a linear model to test the relationship between the tPBM session and the 21 behavioral K value; (2) a generalized linear model was established with “Active vs. Sham” as the predictors, 22 "CDA set-size effect" as the mediator, and "behavioral $\mathrm{K}$ value" as the predicted variable. The direct and 3 indirect effects were then obtained by contrasting these two models. The null hypothesis was tested by 24 examining whether zero was within the $95 \%$ bootstrap confidence intervals (CIs).

We conducted a Bayesian analysis (conducted with JASP software v. 0.13.1.0) to test for evidence for the null. Bayes factor analyses with default priors $(\mathrm{r}=0.707)$ was performed on the EEG data $\left(\mathrm{BF}_{10}=\right.$ support for $\mathrm{H}_{1}$ over $\mathrm{H}_{0} ; \mathrm{BF}_{10}<0.333$ : substantial evidence for the null).

\section{Discussion}

31 Across four complementary EEG experiments, we provided the converging evidence that $1064 \mathrm{~nm}$ tPBM 32 applied to the right PFC could improve visual WM capacity. In the first two experiments, behavioral K values can be enhanced for both orientation and color WM by $1064 \mathrm{~nm}$ tPBM applied on the right PFC. Crucially, we 34 found such WM memory improvements were tracked by individual CDA set-size effects. A mediation analyses 35 revealed that the CDA mediated the behavioral enhancements with tPBM. Further studies demonstrated that 36 effects on capacity enhancement of visual WM was absent for tPBM applied at 852nm tPBM (Experiment 3) 37 and to the left PFC (Experiment 4). 
The reason why $\mathrm{PBM}$ has not been widely adopted to improve WM in humans might be the absence of a

neurophysiological account of tPBM-linked performance gains. In the current study, a well-established

change-detection task allows us to directly estimate visual WM's capacity limits while recording the EEG ${ }^{22}$.

\section{$H 1$}

This allowed us to uncover the link between the behavioral benefits of tPBM and the underlying neuronal mechanism. Another reason might be that tPBM more or less with thermal effect might lead to placebo or subject-expectancy effects towards the active-tPBM could thus be expected, which hard to provide convincing and exclusive evidence for tPBM effect. Thus, we reduced the chosen irradiance of $250 \mathrm{~mW} / \mathrm{cm}^{2}$ previously used to $167 \mathrm{~mW} / \mathrm{cm}^{2}$ in the present study, which just ensure that participants were unable to report which stimulation they had received at either of the two sessions. We also applied double-blind, randomized tPBM protocols to rule out the potential observer-expectancy effects associated with active tPBM.

Based on strictly designed experiments, we provided first evidence that $1064 \mathrm{~nm}$ tPBM applied on right PFC can benefit subsequent behavioral $\mathrm{K}$ value with increasing occipitoparietal CDA set-size effects during the retention. Because the CDA set-size effect reflects the number of objects online-held in visual $\mathrm{WM}^{21}$, our results suggested that $1064 \mathrm{~nm}$ tPBM on right PFC could boost the the visual WM capacity. It corroborates and extends existing findings that active maintenance of visual information in occipitoparietal cortex could be boosted via enhancing the contribution of right PFC in WM maintenance visual WM ${ }^{26}$. Importantly, we established neurophysiological links between the $1064 \mathrm{~nm}$ tPBM and subsequent WM performance, in which CDA during the retention served as a complete mediator. It suggest that increased performance on WM from $1064 \mathrm{~nm}$ tPBM might stem from the right PFC stronger engaging parietal areas as reflected by increase CDA set-size effect. However, given that either hemodynamic activities ${ }^{22,27}$ or $\mathrm{EEG}^{28}$ within the parietal cortex is correlated with WM capacity both between and within subjects, we could not pinpoint whether CDA set-size effect plays a casual role of enhanced WM capacity or being a by-product of hemodynamic activities. Given that the frontoparietal network (FPN) including supplementary motor area (SMA), PFC and IPS is thought to be important for $\mathrm{WM}^{29}$, we suggest that the $1064 \mathrm{~nm}$ tPBM might increase the metabolism (e.g. providing more ATP) in right PFC with positive benefits for the WM-related FPN network. An alternative explanation is that the neurovascular coupling between hemodynamic activities and EEG plays a role in visual information 
34 processing ${ }^{30,31}$. Thus, further EEG-fNIRS/fMRI studies are necessary to gain a better understanding of the

5 underlying mechanism of beneficial effects from tPBM.

Note that tPBM with $852 \mathrm{~nm}$ contained the same laser energy over the same time as $1064 \mathrm{~nm}$ and thus resulted in comparative heating. It can be considered as an active-controlled group to eliminate the exogenous thermal effect that would bias or confound the observed changes. To our knowledge, these results provide the first evidence of wavelength-specific WM capacity improvement by tPBM. Meanwhile, uncertainty remains about the photobiomodulation mechanism at different illumination parameters in the human brain. Further research should determine how variation in illumination parameters, such as power density, treatment timing and pulse structure, would affect the memory-enhancing effects of tPBM.

Our results showed that tPBM contribution to WM capacity is specific to the right PFC rather than the left PFC. It supports that the right PFC was more closely associated with information maintenance in visual-spatial $\mathrm{WM}^{18}$. Given previous work showing that WM capacity could be modulated by increasing the PFC 
39 excitability $^{33}$, we offered a new effective intervention that could enhance visual WM capacity and provided new

30 evidence for a causal relation between right PFC and visual WM capacity ${ }^{34,35}$.

In the past decade, some NIBS technology such as tDCS has been shown to enhance WM performance by increasing the stimulated cortical excitability. The null effect of left PFC stimulation of tPBM in Experiment 4 is consistent with the observation that applying anodal tDCS over the left PFC failed to improve visual WM capacity $^{36}$. However, some other studies have shown that applying anodal tDCS over the left PFC could improve behavioral performance during verbal WM tasks ${ }^{37-39}$. Although both left and right PFC might have general beneficial effects from NIBS technology, as "central executive" is an important unit in the classic storage-and-processing mode of $\mathrm{WM}^{40}$, the recent review pointed out the distinct neural mechanisms between visual and verbal $\mathrm{WM}^{41}$. Anyhow, our observations expand the role of the right PFC in visual WM processing by providing a causal link between behavioral outcomes and tPBM.

Furthermore, anodal tDCS over the right posterior parietal cortex (PPC) could immediately improve WM performance in individuals with low WM capacity ${ }^{4}{ }^{20}$. It suggested that the upper limit to WM in humans cannot be easily broken through tDCS, at least for high-performing individuals who are already above average to begin with. However, our results showed a performance-independent effect (both large and insufficient WM capacity could be improved) across different WM tasks after active $1064 \mathrm{~nm}$ tPBM. We suggested that tPBM is a useful tool for improving WM's upper limits by augmenting the neural metabolism of the relevant frontal regions. Alternatively, recent studies have attempted to improve WM performance and modulate brain activity through within-trial rhythmic entrainment from alternating stimulation ${ }^{2}$ and repetitive magnetic stimulation ${ }^{3}$. These studies suggest that this NIBS technology can bring the peak and online benefit of behavior and neurophysiological gains by modulating temporally neuronal oscillations when administered simultaneously to the WM task. However, participants subjected to $1064 \mathrm{~nm}$-tPBM performed better after the first two blocks than those who received the sham-, $852 \mathrm{~nm}$ - or left-tPBM. It seems that tPBM can yield significant benefits of behavior after several minutes, but not immediately. These observations might stem from tPBM which required the involvement of multi-process of brain activity, unlike that tDCS induce the change of the underlying cortex by causing the neuron's resting membrane potential to depolarize or hyperpolarize, which is consistent with 
15 previous research that $\mathrm{PBM}$ modulate $\mathrm{CCO}$, yield optimal impact when administered after the target task over 16 several minutes ${ }^{42-44}$.

17 In conclusion, our study provides novel and compelling evidence that tPBM can effectively enhance visual I8 working memory capacity in humans. Considering that several diseases, such as attention-deficit hyperactivity, 19 Alzheimer showed a decline in WM capacity, our observations offer an effective, cost-effective, safe and 20 non-invasive brain intervention for future clinical intervention. So far, there are no side effects or harm 21 associated with tPBM reported in the literature, which gives security for issues of safety that will be required. 22 Indeed, the effect of tPBM might depend on the ability of light to penetrate the intracranial depths, as well as 33 the power density, wavelength, and dosage. Further work is needed from biophysical and neurobiological 24 aspects to exploit the full potential of tPBM for healthy and clinical populations. 


\section{References}

1. Jaeggi, S.M., Buschkuehl, M., Jonides, J. \& Perrig, W.J. Improving fluid intelligence with training on working memory. Proc Natl Acad Sci U S A 105, 6829-6833 (2008).

2. Reinhart, R.M.G. \& Nguyen, J.A. Working memory revived in older adults by synchronizing rhythmic brain circuits. Nature neuroscience 22, 820-827 (2019).

3. Albouy, P., Weiss, A., Baillet, S. \& Zatorre, R.J. Selective Entrainment of Theta Oscillations in the Dorsal Stream Causally Enhances Auditory Working Memory Performance. Neuron 94, 193-206 e195 (2017).

4. Tseng, P., et al. Unleashing potential: transcranial direct current stimulation over the right posterior parietal cortex improves change detection in low-performing individuals. The Journal of neuroscience : the official journal of the Society for Neuroscience 32, 10554-10561 (2012).

5. Urquhart, E.L., et al. Transcranial photobiomodulation-induced changes in human brain functional connectivity and network metrics mapped by whole-head functional near-infrared spectroscopy in vivo. Biomedical optics express 11, 5783-5799 (2020).

6. Tian, F., Hase, S.N., Gonzalez-Lima, F. \& Liu, H. Transcranial laser stimulation improves human cerebral oxygenation. Lasers Surg Med 48, 343-349 (2016).

7. Salehpour, F., et al. Brain Photobiomodulation Therapy: a Narrative Review. Mol Neurobiol 55, 6601-6636 (2018).

8. Lampl, Y., et al. Infrared laser therapy for ischemic stroke: a new treatment strategy: results of the NeuroThera Effectiveness and Safety Trial-1 (NEST-1). Stroke 38, 1843-1849 (2007).

9. Nawashiro, H., Wada, K., Nakai, K. \& Sato, S. Focal increase in cerebral blood flow after treatment with near-infrared light to the forehead in a patient in a persistent vegetative state. Photomedicine and laser surgery 30, 231-233 (2012).

10. Rojas, J.C. \& Gonzalez-Lima, F. Neurological and psychological applications of transcranial lasers and LEDs. Biochemical pharmacology 86, 447-457 (2013).

11. Lee, H.I., et al. Pre-conditioning with transcranial low-level light therapy reduces neuroinflammation and protects blood-brain barrier after focal cerebral ischemia in mice. Restor Neurol Neurosci 34, 201-214 (2016). 12. Rojas, J.C., Bruchey, A.K. \& Gonzalez-Lima, F. Low-level light therapy improves cortical metabolic capacity and memory retention. J Alzheimers Dis 32, 741-752 (2012).

13. Michalikova, S., Ennaceur, A., van Rensburg, R. \& Chazot, P.L. Emotional responses and memory performance of middle-aged CD1 mice in a 3D maze: effects of low infrared light. Neurobiol Learn Mem 89, 480-488 (2008).

14. Barrett, D.W. \& Gonzalez-Lima, F. Transcranial infrared laser stimulation produces beneficial cognitive and emotional effects in humans. Neuroscience 230, 13-23 (2013).

15. Vargas, E., et al. Beneficial neurocognitive effects of transcranial laser in older adults. Lasers in medical science 32, 1153-1162 (2017).

16. Blanco, N.J., Maddox, W.T. \& Gonzalez-Lima, F. Improving executive function using transcranial infrared laser stimulation. J Neuropsychol 11, 14-25 (2017).

17. Cowan, N. The magical number 4 in short-term memory: a reconsideration of mental storage capacity. Behav Brain Sci 24, 87-114; discussion 114-185 (2001).

18. Volle, E., et al. Specific cerebral networks for maintenance and response organization within working memory as evidenced by the 'double delay/double response' paradigm. Cereb Cortex 15, 1064-1074 (2005).

19. Vogel, E.K. \& Machizawa, M.G. Neural activity predicts individual differences in visual working memory capacity. Nature 428, 748-751 (2004).

20. Hsu, T.Y., Tseng, P., Liang, W.K., Cheng, S.K. \& Juan, C.H. Transcranial direct current stimulation over right posterior parietal cortex changes prestimulus alpha oscillation in visual short-term memory task. NeuroImage 98, 306-313 (2014).

21. Luria, R., Balaban, H., Awh, E. \& Vogel, E.K. The contralateral delay activity as a neural measure of visual working memory. Neuroscience and biobehavioral reviews 62, 100-108 (2016).

22. Vogel, E.K., McCollough, A.W. \& Machizawa, M.G. Neural measures reveal individual differences in controlling access to working memory. Nature 438, 500-503 (2005). 
23. Wang, X., et al. Up-regulation of cerebral cytochrome-c-oxidase and hemodynamics by transcranial infrared laser stimulation: A broadband near-infrared spectroscopy study. J Cereb Blood Flow Metab 37, 3789-3802 (2017).

24. Li, D., et al. Visual Working Memory Guides Spatial Attention: Evidence from alpha oscillations and sustained potentials. Neuropsychologia 151, 107719 (2021).

25. Pascual-Marqui, R.D., et al. Low resolution brain electromagnetic tomography (LORETA) functional imaging in acute, neuroleptic-naive, first-episode, productive schizophrenia. Psychiatry research 90, 169-179 (1999).

26. Edin, F., et al. Mechanism for top-down control of working memory capacity. Proc Natl Acad Sci U S A 106, 6802-6807 (2009).

27. Fukuda, K. \& Vogel, E.K. Human variation in overriding attentional capture. The Journal of neuroscience : the official journal of the Society for Neuroscience 29, 8726-8733 (2009).

28. Tsubomi, H., Fukuda, K., Watanabe, K. \& Vogel, E.K. Neural limits to representing objects still within view. The Journal of neuroscience : the official journal of the Society for Neuroscience 33, 8257-8263 (2013). 29. Zanto, T.P. \& Gazzaley, A. Fronto-parietal network: flexible hub of cognitive control. Trends in cognitive sciences 17, 602-603 (2013).

30. Zhao, C., et al. Anticipatory alpha oscillation predicts attentional selection and hemodynamic response. Human brain mapping 40, 3606-3619 (2019).

31. Zhao, C., et al. The Neurovascular Couplings Between Electrophysiological and Hemodynamic Activities in Anticipatory Selective Attention. Cereb Cortex (2022).

32. Pruitt, T., et al. Transcranial Photobiomodulation (tPBM) With 1,064-nm Laser to Improve Cerebral Metabolism of the Human Brain In Vivo. Lasers Surg Med 52, 807-813 (2020).

33. Brunoni, A.R. \& Vanderhasselt, M.A. Working memory improvement with non-invasive brain stimulation of the dorsolateral prefrontal cortex: a systematic review and meta-analysis. Brain and cognition 86, 1-9 (2014). 34. Habekost, T. \& Rostrup, E. Visual attention capacity after right hemisphere lesions. Neuropsychologia 45, 1474-1488 (2007).

35. Beck, D.M., Muggleton, N., Walsh, V. \& Lavie, N. Right parietal cortex plays a critical role in change blindness. Cerebral cortex 16, 712-717 (2006).

36. Wang, S., Itthipuripat, S. \& Ku, Y. Electrical Stimulation Over Human Posterior Parietal Cortex Selectively Enhances the Capacity of Visual Short-Term Memory. J Neurosci 39, 528-536 (2019).

37. Fregni, F., et al. Anodal transcranial direct current stimulation of prefrontal cortex enhances working memory. Experimental brain research 166, 23-30 (2005).

38. Ohn, S.H., et al. Time-dependent effect of transcranial direct current stimulation on the enhancement of working memory. Neuroreport 19, 43-47 (2008).

39. Andrews, S.C., Hoy, K.E., Enticott, P.G., Daskalakis, Z.J. \& Fitzgerald, P.B. Improving working memory: the effect of combining cognitive activity and anodal transcranial direct current stimulation to the left dorsolateral prefrontal cortex. Brain Stimul 4, 84-89 (2011).

40. Hitch, G.J. Short-term memory for spatial and temporal information. Q J Exp Psychol 26, 503-513 (1974). 41. van Ede, F. Mnemonic and attentional roles for states of attenuated alpha oscillations in perceptual working memory: a review. The European journal of neuroscience 48, 2509-2515 (2018).

42. Telch, M.J., et al. Effects of post-session administration of methylene blue on fear extinction and contextual memory in adults with claustrophobia. Am J Psychiatry 171, 1091-1098 (2014).

43. Smits, J.A., et al. D-cycloserine enhancement of fear extinction is specific to successful exposure sessions: evidence from the treatment of height phobia. Biological psychiatry 73, 1054-1058 (2013).

44. Rojas, J.C., Bruchey, A.K. \& Gonzalez-Lima, F. Neurometabolic mechanisms for memory enhancement and neuroprotection of methylene blue. Prog Neurobiol 96, 32-45 (2012). 